\title{
ИЗМЕНЕНИЕ МОЩНОСТНЫХ ХАРАКТЕРИСТИК БИОЭЛЕКТРИЧЕСКОЙ АКТИВНОСТИ МОЗГА ЧЕЛОВЕКА ПОД ВЛИЯНИЕМ СПЕЛЕОКЛИМАТОТЕРАПИИ
}

\section{CHANGE OF POWER CHARACTERISTICS OF BIOELECTRIC ACTIVITY OF HUMAN BRAIN UNDER INFLUENCE OF SPELEOCLIMATOTHERAPY}

V. Semiletova

Summary: The purpose of our study is to study changes in the power characteristics of the bioelectric activity of the human brain under the influence of speleoclimatotherapy. The study involved 14 volunteer students, aged 19-22. The electroencephalogram of patients was recorded in a state of functional rest before and after a 2-hour session of speleoclimatherapy using an «Encephalan - EEGR - 19/26» electroencephalograph (Medicom, Taganrog).

Analysis of the average power of brain bioelectric activity of various rhythmic ranges showed that the power of the theta rhythm tended to decrease after caving both with closed and open eyes of the subjects. In a state of functional rest with open and closed eyes, the stress in the central nervous system decreased after exposure to electronegative air ions. The power of the beta- 1 rhythm with closed and open eyes, and the alpha rhythm with open eyes increases after caving. At rest, with closed eyes, the alpha rhythm power decreased slightly in the $\mathrm{C}$ and $\mathrm{C} 4$ regions, the rest-increased. Changes in the power of beta- 2 rhythm are ambiguous. In the functional state of rest with closed eyes, the hemisphere asymmetry of the arising changes manifested itself, on the right, the power of the theta rhythm decreased, on the left, it mainly increased.

The obtained results allow us to recommend speleoclimatotherapy as a method of non-pharmacological correction and prevention of prenosological changes in the health and electrical activity of students' brains, with caution, in a state of health or remission, taking into account the individual approach to each patient and controlling the main parameters of his health during the course of speleoclimatotherapy.

Keywords: speleoclimatotherapy, electroencephalogram, EEG rhythm, brain, bioelectric activity.

\section{Введение}

Спелеоклиматотерапия - моделирование микроклимата сильвинитовых пещер. В настоящее время спелеоклиматотерапия является популярным методом лечения и профилактики различных заболеваний в условиях санаториев, лечебниц и даже поликлиник. Положительный эффект от воздействия микроклимата спелеотерапии объясняют составом воздуха: так, содержание

\section{Семилетова Вера Алексеевна}

К.б.н., дочент, ФГБОУ ВО «Воронежский государственный медицинский университет им. Н.Н. Бурденко»

МинздраваРФ

vera2307@mail.ru

Аннотация: Цель нашего исследования - исследование изменения мощностных характеристик биоэлектрической активности мозга человека под влиянием спелеоклиматотерапии.

Методы. В исследовании приняли участие 14 студентов-добровольцев (1922 года). Электроэнцефалограмма пациентов записана в состоянии функционального покоя до и после 2-х часового сеанса спелеоклиматерапии с помощью электроэнцефалографа “Энцефалан - ЭЭГР - 19/26" фирмы Медиком (г. Таганрог).

Результаты. Анализ средней мощности биоэлектрической активности м0зга различных ритмических диапазонов показал, что мощность тета-ритма имела тенденцию к снижению после спелеосеанса как при закрытых, так и при открытых глазах испытуемых. В состоянии функционального покоя с открытыми и закрытыми глазами у пациентов после воздействия электроотрицательных аэроионов снизилось напряжение в ЦНС.

Мощность ритма бета-1 при закрытых и открытых глазах, и альфа-ритма при открытых глазах увеличивается после спелеосеанса. В состоянии покоя с закрытыми глазами мощность альфа-ритма несколько снизилась в областях Cz и C4, в остальных - возросла. Изменения мощности бета-2 ритма неоднозначны. В функциональном состоянии покоя с закрытыми глазами проявилась межполушарная асимметрия возникающих изменений, справа мощность тета-ритма снизилась, слева - преимущественно увеличилась.

Выводы. Полученные результаты позволяют рекомендовать спелеоклиматотерапию как метод донозологической коррекции и профилактики психосоматического здоровья и ЭЭГ мозга человека, с осторожностью, в состоянии здоровья или ремиссии, учитывая индивидуальный подход к каждому пациенту и контролируя базисные показатели его здоровья во время прохождения курса.

Ключевые слова: спелеоклиматотерапия, электроэнцефалограмма, ритм ЭЭГ, мозг, биоэлектрическая активность.

легких электроотрицательных аэроионов в специально оборудованной спелеокамере составляет 760-960 в $1 \mathrm{~cm}^{3}$, что существенно больше, чем в атмосферном воздухе (содержание электроотрицательных аэроионов в атмосферном воздухе 115-160 в 1 см³) [1].

Согласно литературным данным, под влиянием спелеоклиматотерапии у пациентов наблюдалось повышение физического компонента качества жизни, физиче- 
ского функционирования, интенсивности, психического компонента качества жизни [2].

Исследования показателей иммунного статуса показали увеличение количества Т-лимфоцитов CD45+CD3+, в том числе Т-хелперов CD45+CD3+CD4+, обеспечивающих иммунный ответ крови пациентов под влиянием спелеоклиматотерапии, а также увеличение количества Т-цитотоксических лимфоцитов CD45+CD3+ CD8+, обеспечивающих клеточный иммунитет и В-лимфоцитов CD19+, обеспечивающих гуморальный иммунитет человека [3].

Анализ кардиоритма до и после посещения сеансов спелеоклиматотерапии выявил снижение индекса напряжения, повышение мощности волн спектра HF, что является выражением активности парасимпатического отдела автономной нервной системы и снижение мощности волн спектра VLF, что, как полагают, отражает развитие у пациентов психоэмоционального стресса.

После курса спелеоклиматотерапии наблюдалось снижение показателей ситуативной и личностной тревожности. По параметрам простой зрительно-моторной реакции после прохождения десяти курсов спелеоклиматотерапии наблюдалось достоверное снижение среднего времени реакции, параметры уровня активации центральной нервной системы повышались, а суммарное число ошибок уменьшалось [2].

Следовательно, спелеовоздействие увеличивает адаптационные возможности организма человека благодаря перестройке деятельности нейроиммуноэндокринной системы, что не только препятствует формированию стресс-индуцированных изменений регуляторных систем организма, но и способствует повышению активности иммунной системы и активирует защитные силы организма человека [2].

Несмотря на достаточно подробное изучение общих физиологических эффектов воздействия спелеоклиматотерапии на организм человека, исследований ЭЭГ, на наш взгляд, недостаточно $[4,5]$. А ведь это основная регулирующая система нашего организма, и от ее функционирования будет зависеть работа и иных физиологических органов, и систем.

Цель нашего исследования - исследование изменения мощностных характеристик биоэлектрической активности мозга человека под влиянием спелеоклиматотерапии.

\section{Методы}

В исследовании приняли участие 14 студентов-добровольцев (19-22 года). Исследование проведено на базе кафедры нормальной физиологии ВГМУ им. Н.Н. Бурденко, г. Воронеж. Электроэнцефалограмма пациентов записана в состоянии функционального покоя до и после 2-х часового сеанса спелеоклиматерапии, в малой спелеокамере на одного пациента, с помощью электроэнцефалографа“Энцефалан - ЭЭГР - 19/26" фирмы Медиком (г. Таганрог). Для анализа использовались показатели мощности тета, альфа, бета-1 и бета-2 ритмов в 19-ти зарегистрированных отведениях. Вычисление средних значений, стандартного отклонения, ошибок средних значений и корреляционный анализ проведены с использованием пакета программ Excel и Statistica 13.3.

Среднюю мощность биоэлектрической активности мозга различных ритмических диапазонов мы использовали как интегральный показатель.

Сумма корреляционных связей, учитывая модули представленных связей между признаками, позволила сделать вывод об уровне напряжения в регулирующей системе. Динамика мощностей отдельных ритмов ЭЭГ по отдельным отведениям до и после сеанса спелеоклиматотерапии оценена на основании средних значений мощностей ритмов ЭЭГ по отдельным отведениям. Для наглядности эта динамика представлена в виде рисунка, где разными цветами мы указали уменьшение, увеличение, либо отсутствие изменений в динамике мощности. Сами средние значения не приведены из соображений загруженности статьи и сложности ее восприятия.

\section{Результаты}

Среднюю мощность биоэлектрической активности мозга различных ритмических диапазонов до и после влияния спелеосеанса рассчитали как среднее значение мощностей отдельных ритмов от 19-ти отведений ЭЭГ в целом по группе. Анализ средней мощности биоэлектрической активности мозга различных ритмических диапазонов показал, что мощность тета-ритма имела тенденцию к снижению после спелеосеанса как при закрытых ( $=0,704)$, так и при открытых глазах испытуемых $(p=0,844)$.

Мощность альфа и бета-1 ритмов достоверно возрастала закрытых ( $p=0.0004$ и $p=0)$ при открытых глазах $(p=0,001)$ испытуемых. Мощность бета-2 ритмов при открытых глазах пациентов не изменялась $(p=0,704)$, при закрытых глазах - имела тенденцию к повышению $(p=0,203)$, табл. 1.

Проведен корреляционный анализ для оценки взаимосвязи средней мощности биоэлектрической активности мозга различных ритмических диапазонов до и после влияния сеанса спелеоклиматотерапии, рис 2. Достоверность корреляционных связей не рассчитывалась, поскольку мы использовали корреляционный ана- 
Таблица 1.

Средняя мощность биоэлектрической активности мозга различных ритмических диапазонов до и после влияния спелеосеанса

\begin{tabular}{|l|c|c|c|c|c|c|}
\hline & Среднее, до & СК0, до & Ошибка среднего, 0Г & Среднее, после & СК0, после & Ошибка среднего, после \\
\hline Тета-ритм, ОГ & 7,056 & 1,491 & 0,107 & 6,891 & 1,806 & 0,129 \\
\hline Тета-ритм, 3Г & 7,269 & 4,024 & 0,287 & 7,080 & 1,896 & 0,135 \\
\hline Альфа-ритм, 0Г & 9,525 & 5,023 & 0,359 & 13,399 & 9,881 & 0,706 \\
\hline Альфа-ритм, 3Г & 17,316 & 9,639 & 0,688 & 20,631 & 11,936 & 0,853 \\
\hline Бета-1 ритм, 0Г & 7,971 & 2,095 & 0,150 & 15,597 & 4,067 & 0,291 \\
\hline Бета-1 ритм, 3Г & 8,182 & 1,784 & 0,127 & 42,363 & 8,612 & 0,615 \\
\hline Бета-2 ритм, ОГ & 4,267 & 2,021 & 0,144 & 4,036 & 1,562 & 0,112 \\
\hline Бета-2 ритм, ЗГ & 3,826 & 1,088 & 0,078 & 4,359 & 2,240 & 0,160 \\
\hline
\end{tabular}
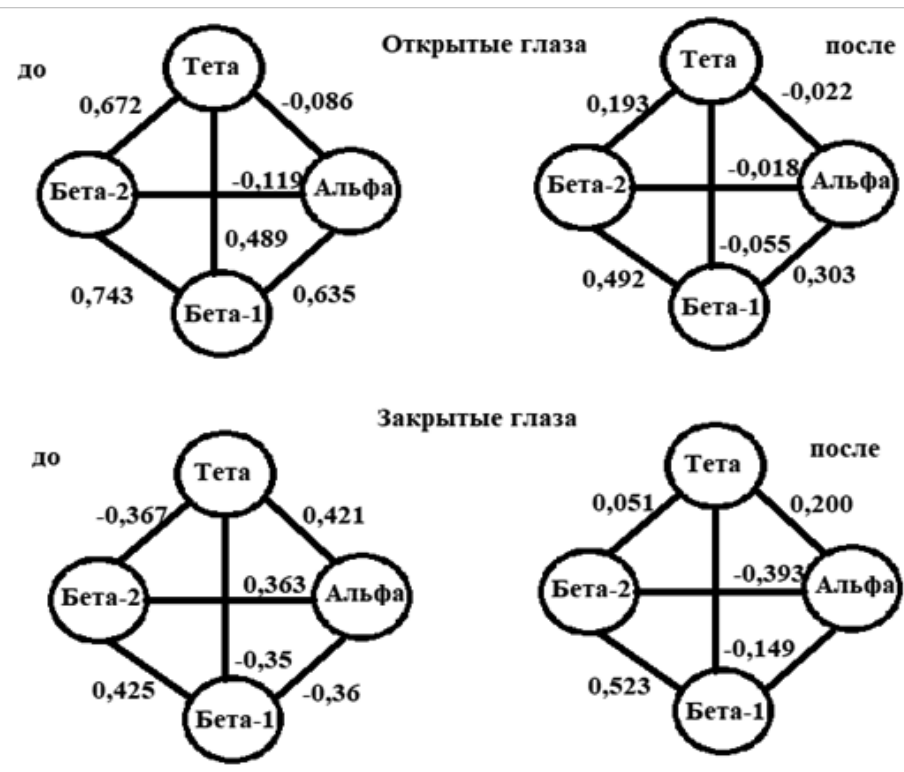

Закрытые глаза

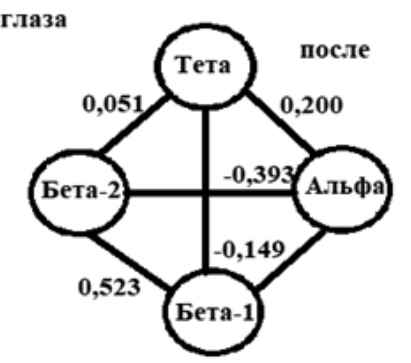

Рис. 1. Корреляционные связи средней мощности биоэлектрической активности мозга различных ритмических диапазонов до и после влияния сеанса спелеоклиматотерапии

лиз как маркер напряженности регулирующей системы организма.

Проведенный корреляционный анализ показал, что в функциональном состоянии покоя при открытых глазах происходит ослабление связи между изучаемыми мощностными характеристиками (например, Альфа-Бета-1 равно 0,635 до и 0,303 после: Бета-1-Бета-2 равно 0,743 до и 0,492 после).

Для наглядности мы рассчитали сумму корреляционных связей, учитывая модули представленных связей между признаками. В состоянии покоя с открытыми глазами сумма составила 2,743, после спелеосеанса 1,083. Известно, что чем сильнее взаимосвязи между признаками в системе, тем выше напряжение в системе. В нашем случае, у пациентов в состоянии покоя с открытыми глазами после воздействия электроотрицательных аэроионов напряжение в ЦНС снизилось.

Несколько сложнее наблюдаемые изменения у пациентов в состоянии покоя с закрытыми глазами. Сумма корреляционных связей, учитывая модули представленных связей между признаками, составила 2,286 до и 1,356 после спелеосеанса. Однако обращает на себя внимание инверсия знаков корреляционных связей между мощностями тета и бета-2 ритмов $(-0,367$ и 0,051) и альфа и бета-2 ритмов (0,363 и -0,393), а также увеличение корреляционной связи между бета-1 и бета-2 $(0,425$ и 0,523) диапазонами ЭЭГ.

Поскольку средняя мощность биоэлектрической активности мозга различных ритмических диапазонов отражает только общие изменения ЭЭГ после сеанса спелеоклиматотерапии, мы распределили испытуемых в зависимости от динамики: с повышением, понижением 


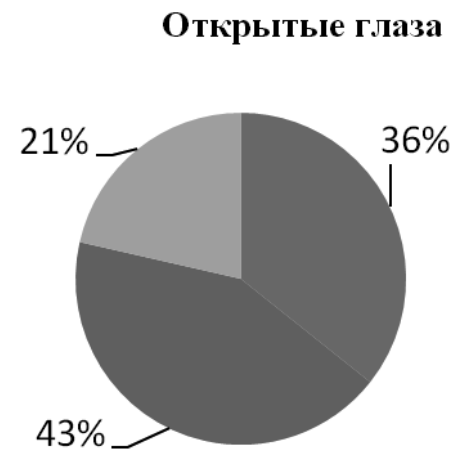

Тета

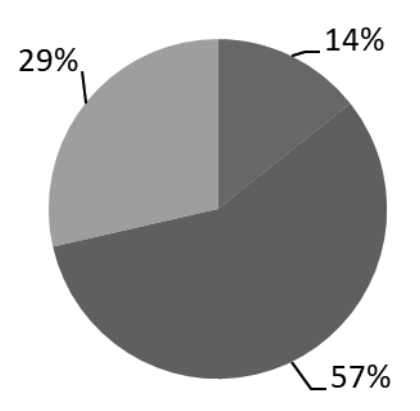

Альфа

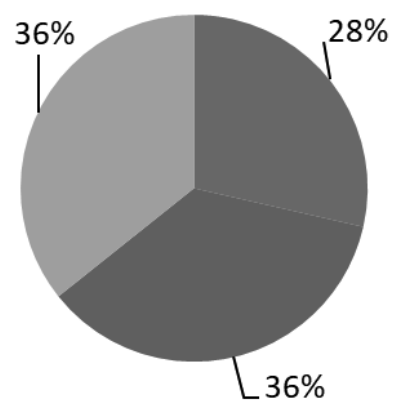

Бета-1

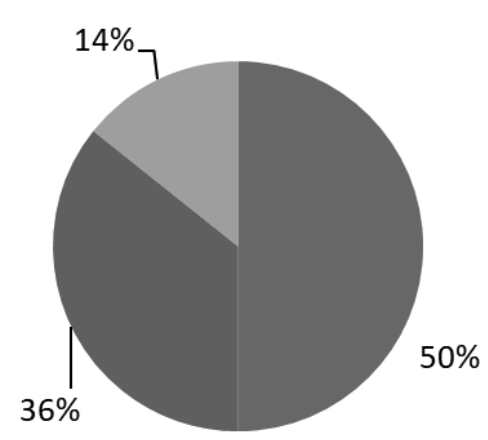

Бета-2
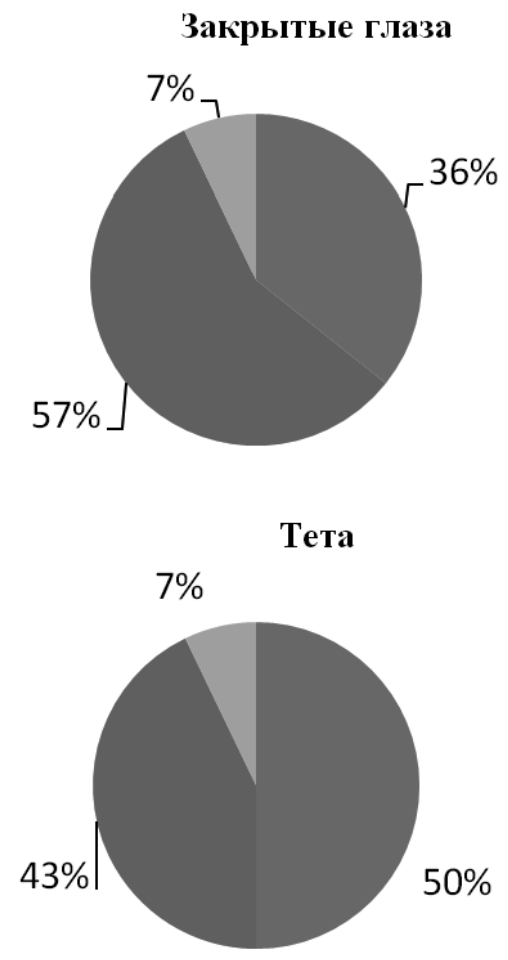

Альфа

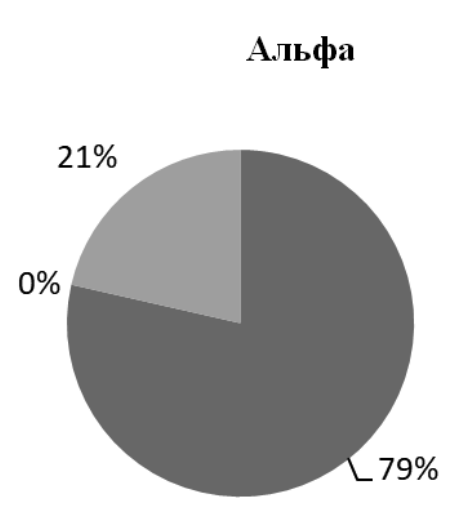

Бета-1

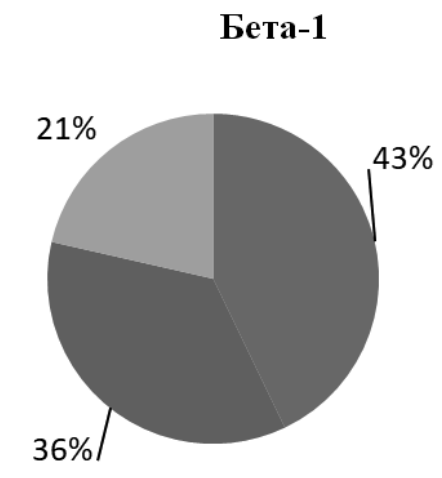

повышение ппонение

Бета-2

Рис. 2. Распределение испытуемых в зависимости от динамики мощности после сеанса спелеоклиматотерапии. 
мощности и отсутствием изменений, рис. 1.

Выявлено, что спелеоклиматотерапия обладает индивидуальным, неоднозначным воздействием на мощность ЭЭГ ритмов. Так, в состоянии покоя с открытыми глазами у 43\% испытуемых повышается мощность тетаритма, у 57\% - мощность альфа-ритма, у 36\% - мощность бета-1 и бета-2 ритмов. В состоянии покоя с закрытыми глазами динамика мощности альфа, бета-1 и бета-2 ритмов у большинства испытуемых имеет тенденцию к снижению; динамика мощности тета-ритма - к повышению, рис. 2.

Поэтому, поскольку речь идет о здоровье каждого пациента, - следует учитывать индивидуальные изменения ЭЭГ пациента после воздействия спелеотерапии, и глобальной актуальной проблемой встает изучение тонких ЭЭГ-механизмов этого воздействия. Далее мы провели анализ динамики мощностей отдельных ритмов ЭЭГ до и после сеанса спелеоклиматотерапии, результаты представлены на рис. 3. Отметим, что изменения бета1 диапазона ритмов ЭЭГ однозначны как при закрытых, так и при открытых глазах, мощность ритма бета-1 увеличивается.

Мощность альфа-ритма также увеличилась при открытых глазах генерализованно, независимо от области головного мозга. В состоянии покоя с закрытыми глазами мощность альфа-ритма несколько снизилась в областях $C_{z}$ и $C_{4}\left(C_{z}: 31,74 \pm 1,655\right.$ и 30,62 $\pm 1,29 ; C_{4}: 23,93 \pm 1,309$

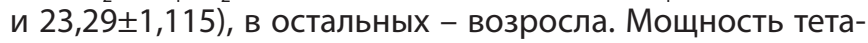
ритма в состоянии функционального покоя с открытыми глазами увеличилась во фронтальных и центральных, и снизилась в затылочных, теменных и височных областях мозга.

В функциональном состоянии покоя с закрытыми глазами проявилась межполушарная асимметрия возникающих изменений, справа мощность тета-ритма снизилась, слева - преимущественно увеличилась.

Наиболее неоднозначно проявляются изменения бета-2 ритма.

В состоянии функционального покоя с открытыми глазами справа в височных областях мощность бета-2 ритма увеличилась $\left(\mathrm{T}_{4}\right.$ и $\left.\mathrm{T}_{6}\right)$, слева - снизилась $\left(\mathrm{T}_{3}\right)$. В затылочной области справа мощность не изменилась $\left(\mathrm{O}_{2}\right.$, $4,99 \pm 0,205$ и 5,29 $\pm 0,201)$, слева - возросла $\left(0_{1}, 7,16 \pm 0,277\right.$ и 5,12 00,184$)$. В центральных областях выявлена тенденция к снижению бета-2 активности. Во фронтальных отведениях справа и слева отметили увеличение мощности, ближе к центру - снижение.

В состоянии функционального покоя с закрытыми глазами в затылочных, теменных и центральных $\left(C_{3}\right.$ и $\left.C_{4}\right)$ и $\mathrm{F}_{3}$ областях коры больших полушарий мощность бета-2 ритма возрастает, во фронтальных $\left(\mathrm{F}_{7^{\prime}}, \mathrm{F}_{z^{\prime}} \mathrm{F}_{4^{\prime}} \mathrm{F}_{8}\right)$ - снижается.

\section{Обсужмение результатов}

Таким образом, выявлены изменения биоэлектрической активности мозга под влиянием спелеоклиматотерапевтического сеанса и отмечены индивидуальные особенности изменения мощности. Делая акцент на функциональных особенностях каждого ритма ЭЭГ, следует отметить, что генерализованное повышение альфа ритма может быть связано с отдыхом, состоянием покоя испытуемых.

Тета-ритм исследователи связывают эмоциональным напряжением, стрессом, самообучением, состоянием озарения, качественным усвоением информации. Снижение тета ритма в затылочных, височных и теменных областях может быть связано со снижением стрессорной нагрузки, со снижением напряжения в регуляторных системах под воздействием сеанса спелеотерапии. Увеличение тета-ритма во фронтальных и центральных областях коры может свидетельствовать об активной мыслительной деятельности, принятии решения, озарении.

Повышение бета-1 ритма может быть обусловлено восстановлением работоспособности, готовностью к активной умственной деятельности под влиянием спелеосенса.

Межполушарная асимметрия изменения тета-ритма при закрытых глазах отражает увеличение аналитической стратегии переработки информации и доли произвольной регуляции с участием сознания.

Таким образом, возвращаясь к интегральным показателям, следует отметить, что среднюю мощность биоэлектрической активности мозга различных ритмических диапазонов можно использовать как простейший индикатор изменения уровня функциональной активности ЭЭГ пациента (пациентов) под воздействием аэроионов. Корреляционный анализ средних мощностей биоэлектрической активности мозга различных ритмических диапазонов до и после влияния сеанса спелеоклиматотерапии отражают уровень напряжения в регуляторной системе, и его снижение характеризует спелеовоздействие как положительное.

Однако, на наш взгляд, объяснения влияния сеанса спелеоклиматотерапии на ЭЭГ человека с позиций функций головного мозга являются недостаточными. Высокое содержание электроотрицательных аэроионов в воздухе спелеокамеры однозначно оказывают влияние на функционирование отдельных нервных клеток. 


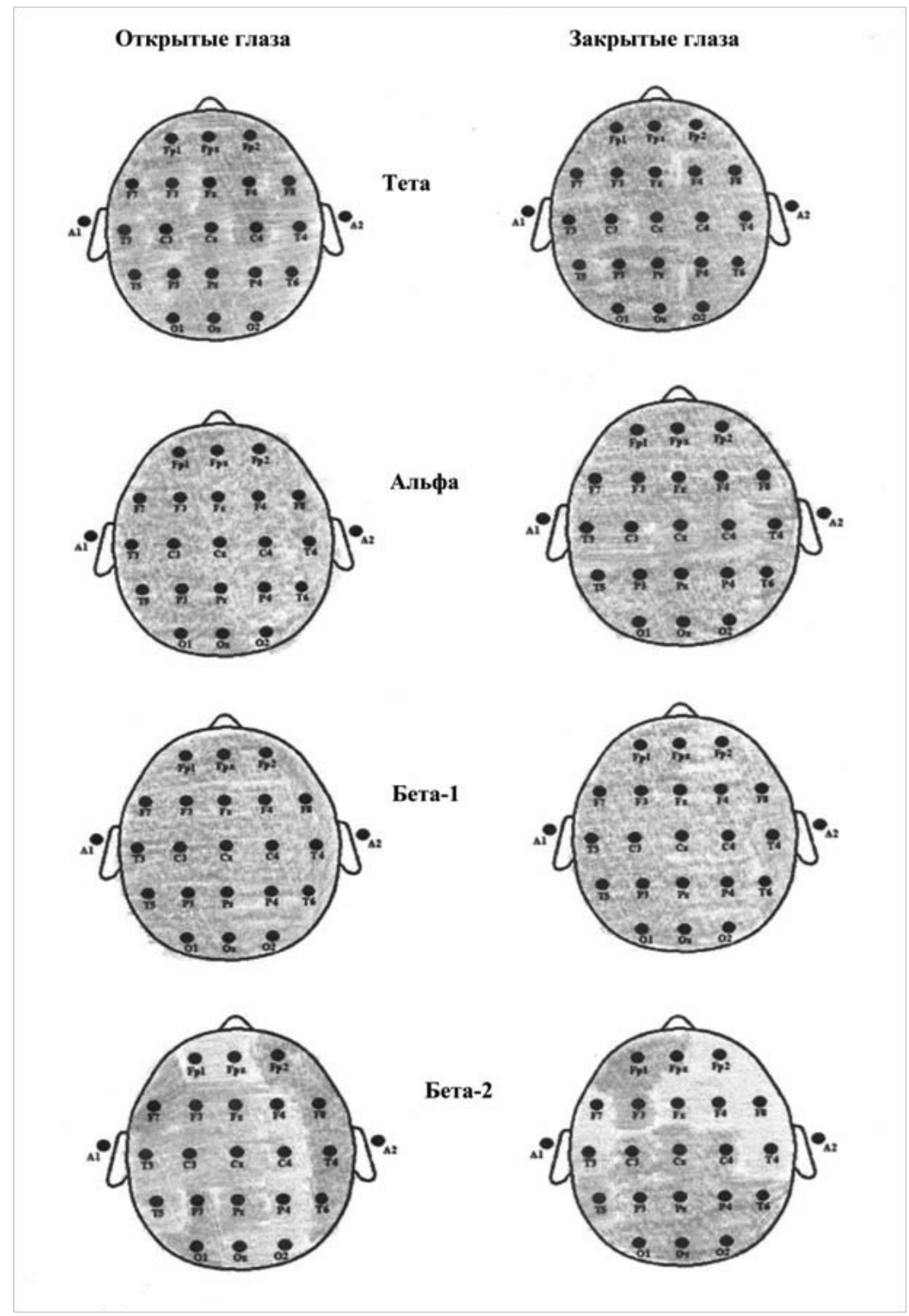

Рис. 3. Анализ динамики мощностей отдельных ритмов ЭЭГ до и после сеанса спелеоклиматотерапии.

Обращаясь к механизмам формирования потенциалов действия нервных клеток, можно утверждать, что изменение содержания $\mathrm{Na}^{+}, \mathrm{K}^{+}, \mathrm{Cl}^{-}$неизбежно приведет к изменению механизмов формирования потенциалов действия. И это тоже будет отражаться в функциях коры больших полушарий головного мозга.

Следовательно, проводя лечебные воздействия в условиях сильвинитовой спелеокамеры, следует помнить об индивидуальных особенностях воздействия аэроионов на ЭЭГ человека. Поэтому требуется детальное изучение тонких физиологических механизмов изменения биоэлектрической активности мозга.

Полученные результаты позволяют рекомендовать спелеоклиматотерапию как метод донозологической коррекции и профилактики психосоматического здоровья и ЭЭГ мозга человека, с осторожностью, в состоянии здоровья или ремиссии, учитывая индивидуальный подход к каждому пациенту и контролируя базисные показатели его здоровья во время прохождения курса.

\section{Зак^ючения (выводы)}

1. Анализ средней мощности биоэлектрической активности мозга различных ритмических диапазонов показал, что мощность тета-ритма имела тенденцию к снижению после спелеосеанса как при закрытых, так и при открытых глазах испытуемых.

2. В состоянии функционального покоя с открытыми и закрытыми глазами у пациентов после воздействия электроотрицательных аэроионов снизи- 
лось напряжение в ЦНС.

3. Мощность ритма бета-1 при закрытых и открытых глазах, и альфа-ритма при открытых глазах увеличивается после спелеосеанса. В состоянии покоя с закрытыми глазами мощность альфа-ритма несколько снизилась в областях $\mathrm{C}_{z}$ и $\mathrm{C}_{4}\left(\mathrm{C}_{\mathrm{z}}: 31,74 \pm 1,655\right.$ и 30,62 $\pm 1,29 ; \mathrm{C}_{4}: 23,93 \pm 1,309$ и $23,29 \pm 1,115)$, в остальных - возросла. Изменения мощности бета-2 ритма неоднозначны.

4. Мощность тета-ритма в состоянии функционального покоя с открытыми глазами увеличилась во фронтальных и центральных, и снизилась в затылочных, теменных и височных областях мозга. В функциональном состоянии покоя с закрытыми глазами проявилась межполушарная асимметрия возникающих изменений, справа мощность тетаритма снизилась, слева - преимущественно уве- личилась.

5. Проводя лечебные воздействия в условиях сильвинитовой спелеокамеры, следует помнить об индивидуальных особенностях воздействия аэроионов на ЭЭГ человека. Поэтому требуется детальное изучение тонких физиологических механизмов изменения биоэлектрической активности мозга.

6. Полученные результаты позволяют рекомендовать спелеоклиматотерапию как метод донозологической коррекции и профилактики психосоматического здоровья и ЭЭГ мозга человека, с осторожностью, в состоянии здоровья или ремиссии, учитывая индивидуальный подход к каждому пациенту и контролируя базисные показатели его здоровья во время прохождения курса.

\section{ЛИТЕРАТУРА}

1. Файнбург Г.З. 0 доказательности эффективности методов спелеотерапии в калийных рудниках и спелеоклиматотерапии в сильвинитовых спелеокамерах // В сборнике: Актуальные проблемы охраны труда и безопасности производства, добычи и использования калийно-магниевых солей Материалы I Международной научно-практической конференции. Под ред. Г.З. Файнбурга. 2018. С. 416-441.

2. Есауленко И.Э., Дорохов Е.В., Горбатенко Н.П. и др. Эффективность спелеоклиматотерапии у студентов в состоянии хрогического стресса // Экология человека. 2015. № 7. С. 50-57.

3. Дорохов Е.В., Жоголева 0.А. Спелеоклиматотерапия как инновационный метод здоровьесбережения // Вестник новых медицинских технологий. 2011. Т. 18. № 2. С. 133-135.

4. Горбатенко Н.П., Семилетова В.А., Дорохов Е.В. Влияние спелеоклиматотерапии на психоэмоциональное состояние и электрической активность мозга здорового человека // В сборнике: Материалы XXIII съезда Физиологического общества им. И. П. Павлова с международным участием 2017. C. 1145-1147.

5. Невзоров А.Ю., Мухина М.Ю. Спелеотерапия как разновидность альтернативной медицины // Бюллетень медицинских интернет-конференций. 2013. T. 3. № 2. C. 177.

(с) Семилетова Вера Алексеевна (vera2307@mail.ru).

Журнал «Современная наука: актуальные проблемы теории и практики» 